\title{
Effect of particle properties of powders on the generation and transmission of Raman scattering
}

\author{
Nichola Townshend, ${ }^{1}$ Alison Nordon, ${ }^{* 1}$ David Littlejohn, ${ }^{* 1}$ John Andrews ${ }^{2}$ and Paul \\ Dallin $^{2}$ \\ ${ }^{1}$ WestCHEM, Department of Pure and Applied Chemistry and CPACT, University of \\ Strathclyde, 295 Cathedral Street, Glasgow, G1 1XL, UK \\ 2 Clairet Scientific Ltd., 17/18 Scirocco Close, Moulton Park Industrial Estate, \\ Northampton, NN3 6AP, UK \\ * denotes authors to whom correspondence should be sent \\ (Email: d.littlejohn@strath.ac.uk and alison.nordon@strath.ac.uk)
}

\begin{abstract}
Transmission Raman measurements of a $1 \mathrm{~mm}$ thick sulphur-containing disc were made at different positions as it was moved through $4 \mathrm{~mm}$ of aspirin $(150-212 \mu \mathrm{m})$ or microcrystalline cellulose (Avicel) of different size ranges $(<38,53-106$ and $150-$ $212 \mu \mathrm{m})$. The transmission Raman intensity of the sulphur inter-layer at $218 \mathrm{~cm}^{-1}$ was lower when the disc was placed at the top or bottom of the powder bed, compared to positions within the bed and the difference between the sulphur intensity at the outer and inner positions increased with Avicel particle size. Also, the positional intensity difference was smaller for needle-shaped aspirin than for granular Avicel of the same size. The attenuation coefficients for the propagation of the exciting laser and transmitted Raman photons through the individual powders were the same, but decreased as the particle size of Avicel increased; also, the attenuation coefficients for propagation through $150-212 \mu \mathrm{m}$ aspirin were almost half of those through similar sized Avicel particles. The study has demonstrated that particulate size and type affect transmitted Raman intensities and consequently, such factors need to be considered in the analysis of powders especially if particle properties vary between the samples.
\end{abstract}




\section{Introduction}

Recently, there has been increasing interest in the use of transmission Raman spectrometry, rather than the backscatter method, for the analysis of pharmaceutical dosage forms. ${ }^{1-10}$ This is because transmission Raman spectrometry has been shown to provide a measure of the bulk composition of a sample, unlike backscatter measurements which exhibit a strong bias towards the upper surface layers. ${ }^{1}$ Consequently, transmission Raman spectrometry can yield more accurate quantification of an active pharmaceutical ingredient (API) in pharmaceutical tablets and capsules. ${ }^{3}$ In addition, it has been shown that simpler and more robust multivariate calibration models can be employed when spectra are collected in the transmission mode. ${ }^{3,8}$ Raman or fluorescence signals from the surface of samples such as tablet coatings or capsule shells, which dominate backscatter Raman measurements, are also significantly reduced in transmission spectra. ${ }^{2,4}$

Although transmission Raman spectrometry has not been used extensively to date, the transmission mode is not a recent development in the field of Raman spectrometry. In a number of early studies, the fundamental propagation of laser and Raman photons through solid samples was investigated. ${ }^{11-13}$ In one such study, the propagation of laser and Raman photons through samples with different scattering properties and of varying thickness was calculated. ${ }^{11}$ The transmitted laser intensity decreased with sample thickness whereas a maximum was observed in a plot of transmitted Raman intensity against sample thickness. Transmitted laser and Raman intensities were also measured experimentally through urea and sodium nitrate discs of different thicknesses, ${ }^{12}$ with the trends observed comparable to those predicted by calculation. ${ }^{11}$ A maximum was also observed in a plot of transmitted Raman intensity against sample thickness for various powders ${ }^{13-14}$ and propranolol-mannitol tablets. ${ }^{3}$ In the study by Eremeeva and Vorsina, ${ }^{13}$ the sample thickness, at which the maximum transmitted Raman intensity was observed, increased with particle size, occurring at $1 \mathrm{~mm}$ for particles $<0.125 \mathrm{~mm}$ and at $2 \mathrm{~mm}$ for larger particles. The variation of transmitted Raman intensity with sample thickness can be rationalised by considering the amount of material irradiated by the laser, and thus the intensity of the Raman signal generated, against the probability of the Raman signal reaching the detector. ${ }^{3}$ More recently, the decay of the exciting laser and Raman signal in 
powder samples consisting of a single material have been investigated using numerical simulations and time-resolved measurements in backscatter ${ }^{15-16}$ and transmission ${ }^{17}$ configurations. For both measurement modes, the Raman signal was found to decay slower than the exciting laser intensity, which can be explained on the basis that the laser photons undergo diffuse scattering while Raman photons are generated as well as being diffusely scattered.

A number of studies have been carried out to understand how layers at different depths within a sample contribute to the overall signal measured in the transmission and backscatter modes. Matousek and Parker compared the suitability of backscatter and transmission Raman spectrometry for analysis of the bulk composition of a two-layer sample, which consisted of a $3.9 \mathrm{~mm}$ thick paracetamol tablet and a $2 \mathrm{~mm}$ thick layer of powdered trans-stilbene. ${ }^{1}$ The study showed that irrespective of the orientation of the two-layer sample, peaks of similar intensity were detected for both materials in the transmission spectra. This, however, was not shown to be the case with the equivalent backscatter Raman measurements, where only peaks of the material present on the top surface of the sample were identified in the spectra owing to the strong bias of the backscatter measurement mode towards the surface layers. Sparén et al. carried out a comparative study of the two types of Raman measurements with a two-layer tablet of propranolol and mannitol, ${ }^{6}$ and obtained similar findings to Matousek and Parker. ${ }^{1}$

Matousek and Parker also carried out Monte-Carlo calculations to simulate the Raman signal from a $0.5 \mathrm{~mm}$ thick inter-layer as it was moved through a bulk medium; the total sample thickness was $4 \mathrm{~mm} .{ }^{1}$ In the backscatter mode, the simulations revealed that when the inter-layer was at a depth of $1.5 \mathrm{~mm}$, the intensity was only $3 \%$ of that obtained when the layer was at the surface. Conversely, the corresponding transmission mode results showed that between depths of 0 and $3.5 \mathrm{~mm}$, the Raman inter-layer signal varied by only a factor of 2. More recent Monte Carlo simulations ${ }^{17-19}$ of the transmitted Raman signal showed a stronger bias towards the centre of the sample than predicted by the earlier study. ${ }^{1}$ In all cases, the reduction in signal observed when the inter-layer was located at the sample surface was attributed to loss of laser and Raman photons at the airsample interface. The relative insensitivity of the transmission Raman signal to the position of an inter-layer within a bulk sample was demonstrated experimentally for a 
$0.9 \mathrm{~mm}$ layer of poly(ethyleneterephthalate) (PET) film placed at four different depths within a stack of three microcrystalline cellulose (MCC) discs. ${ }^{19}$ The signal from the inter-layer, PET, was stronger when the PET film was placed within the stack of MCC discs compared to when it was on the surface (upper or lower) owing to photon losses at the air-sample interface. The variation in inter-layer signal with depth was recently investigated at a higher spatial resolution by placing a layer of PET foil (330 $\mu \mathrm{m}$ thick) doped with $\mathrm{TiO}_{2}$ at different positions within eight $0.5 \mathrm{~mm}$ discs of MCC. ${ }^{18}$ The transmitted Raman signal for $\mathrm{TiO}_{2}$ was lower when the inter-layer was placed at the surface of the MCC stack and was relatively insensitive to position when the inter-layer was positioned within the stack; Monte Carlo simulations predicted a stronger dependence on position. The plot of $\mathrm{TiO}_{2}$ signal intensity against depth was symmetrical about the centre of the sample; numerical simulations showed that this should be the case if the level of absorption of the laser and Raman photons is identical. ${ }^{18}$ It was also shown that use of a photon diode at the sample surfaces can reduce the signal bias towards the centre of the sample and simultaneously improve the signal-to-noise ratio of transmission Raman spectra. ${ }^{18}$ From these numerical ${ }^{1,18}$ and experimental ${ }^{1,6,18-19}$ findings, it can be concluded that transmission Raman measurements provide a significantly more representative way of probing the body of a sample compared to the conventional backscatter Raman approach.

While early theoretical and experimental studies of transmission Raman spectrometry considered the effect of the optical properties of a powder on the propagation of laser and Raman photons, ${ }^{11-14}$ such factors have not been considered in recent research examining the contribution of layers at different depths to the overall transmission Raman spectrum. ${ }^{1,18-19}$ This study addresses the issue and reports for the first time the effect that differences in the size and type of particles in a sample have on the transmitted Raman intensity of an inter-layer positioned at different depths. In addition, through comparison of measurements obtained from different experimental configurations, it has been possible to calculate attenuation coefficients associated with the propagation of the incident laser and resultant Raman radiation through the powders. 


\section{Experimental}

\section{Raman spectrometer}

A Kaiser Raman Workstation and PhAT probe (Kaiser Optical systems, Ann Arbor, USA) were used to investigate the effect of inter-layer position on the transmission Raman spectrum and the propagation of Raman photons in Avicel and aspirin. An Invictus diode laser was employed (785 nm, $400 \mathrm{~mW})$. Samples were illuminated from below using a laser spot that had been expanded to $3 \mathrm{~mm}$ diameter and transmitted Raman photons were collected from a $3 \mathrm{~mm}$ diameter circular region on the upper surface of the sample directly above the illumination point. Raman spectra were obtained with an exposure time of $3 \mathrm{~s}$ and 30 accumulations. A dark current spectrum was subtracted from each sample spectrum. Spectra were acquired using HoloGRAMS software and exported as .SPC files into Matlab version 7.0.2 (Mathworks Inc., Natick, Massachusetts, USA) for analysis using PLS_Toolbox version 3.0.4 (Eigenvector Research Inc., Manson, Washington, USA). Second derivative spectra were calculated using the Savitzky-Golay function with a 51 point filter width and second order polynomial.

A second analyser with a different configuration was also used to investigate the propagation of laser and Raman photons in Avicel with a particle size of $150-212 \mu \mathrm{m}$. The laser (785 nm, $400 \mathrm{~mW})$ of a Kaiser RXN1 Raman spectrometer was focussed onto the sample from above using a Kaiser MkII probe head, equipped with a non-contact optic (focal length approx. $6 \mathrm{~cm}$, laser spot size of $80 \mu \mathrm{m}$ ). The transmitted Raman signal was collected from beneath the sample using a Kaiser PhAT probe (with a $6 \mathrm{~mm}$ diameter illumination optic) connected to the RXN1 spectrometer. Raman spectra were acquired with an exposure time of $5 \mathrm{~s}$ and 32 accumulations. A dark current spectrum was subtracted from all spectra. Spectra were recorded using IC Raman software (Mettler-Toledo, Columbus, USA) and exported as individual .SPC files to Matlab version R2007b for analysis using the PLS_Toolbox version 4.1. Second derivative Raman spectra were calculated using the Savitzky-Golay method with a filter width of 11 points and a second order polynomial. 


\section{Materials}

The powders used were Avicel microcrystalline cellulose (particle sizes $0-38,53$ - 106 and $150-212 \mu \mathrm{m})$ donated by GlaxoSmithKline and aspirin $(150-212 \mu \mathrm{m})$ supplied by Sigma Aldrich, UK. The supplied materials were sieved to obtain the desired particle size ranges. ${ }^{20}$ Avicel particles are granular, whereas aspirin is needle-shaped; digital microscope images of the powders were used to give a visual comparison of the size and shape of the sieved material. The Raman active inter-layer was a $1 \mathrm{~mm}$ thick pressed disc of a 50:50 mixture of potassium bromide and flowers of sulphur. The disc was prepared in a conventional disc press operated to a pressure of 25 tonnes.

Flowers of sulphur was chosen as it is a highly efficient Raman scatterer. Underivatised and part of the $2^{\text {nd }}$ derivative transmission Raman spectra of flowers of sulphur, Avicel and aspirin are given in Figures S-1 and S-2, respectively, of the Supporting Information. The $2^{\text {nd }}$ derivative spectrum shows that there is a strong contribution from flowers of sulphur at approximately $218 \mathrm{~cm}^{-1}$ with no interfering contribution from the other compounds. Therefore, the magnitude of this signal was used to investigate photon propagation in Avicel and aspirin.

\section{Methods}

Figure 1a shows the sample configurations, denoted P1 to P5, used to evaluate the effect of the location of the Raman active inter-layer (sulphur disc) within the powders on the transmitted sulphur signal. The powder and disc were supported using a glass slide attached to the underside of a series of $7 \mathrm{~cm} \times 8 \mathrm{~cm}$ stainless steel plates with a $3 \mathrm{~cm}$ diameter circular hole through which the laser radiation could penetrate. The selected powder was carefully placed into the void to the required depths shown in Figure 1a and was levelled off using a razor blade. The disc containing flowers of sulphur was placed at the required position with additional powder placed on top to the required depth. The stack of stainless steel plates containing the powder and disc was then placed into the Kaiser Raman Workstation such that the laser light passed through the centre of the sample held within the stack of plates (illumination from below). 
a)

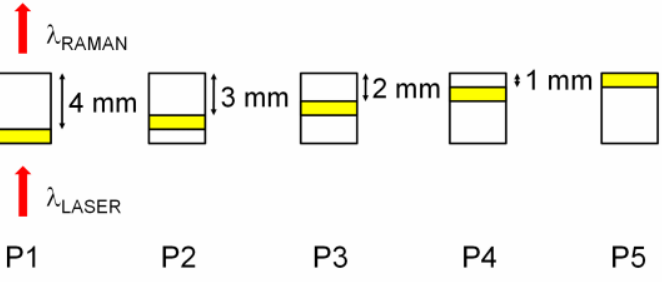

b) $\lambda_{\text {RAMAN }}$

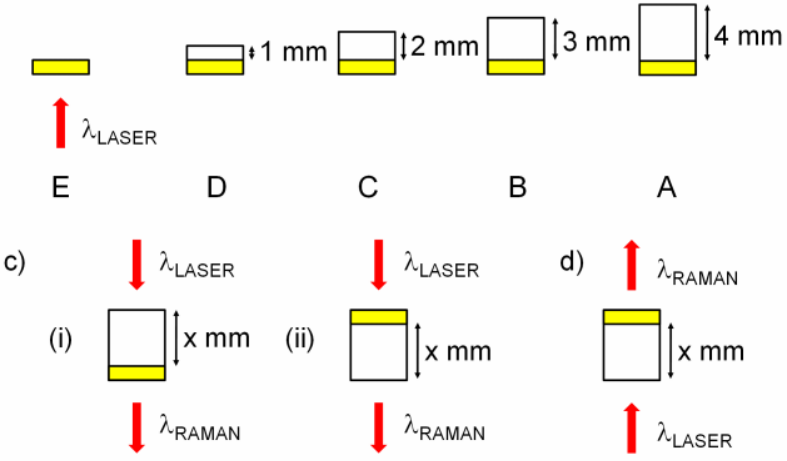

Figure 1. Sample configurations for transmission Raman measurements of a $1 \mathrm{~mm}$ disc containing flowers of sulphur (yellow): a) located at different depths within a bed of powder (white), depth of bed of powder $=4 \mathrm{~mm}$, sample illumination from below; $b$ ) when different depths of powder ( 0 to $4 \mathrm{~mm}$ ) are placed on top of the disc, sample illumination from below; c) when different depths of powder ( $\mathrm{x}=0$ to 6$)$ are placed $\mathrm{i})$ above and ii) below the disc, sample illumination from above; and d) when different depths of powder ( $\mathrm{x}=0$ to 4$)$ are placed below the disc, sample illumination from below. 
The sample configurations denoted A to $\mathrm{E}$ in Figure $1 \mathrm{~b}$ were used with the Kaiser Raman Workstation (illumination from below) to investigate the effect of the different powders, at depths up to $4 \mathrm{~mm}$, on the attenuation of the Raman signal generated by the sulphur disc. The sample configurations shown in (i) and (ii) of Figure 1c were used with the combined Kaiser MkII probe head and PhAT probe set-up (illumination from above) to investigate the attenuation of the laser and Raman photons, respectively, in Avicel with a particle size of $150-212 \mu \mathrm{m}$ at depths up to $6 \mathrm{~mm}$.

The repeatability of measurements at $218 \mathrm{~cm}^{-1}$ made with configurations A and D in Figure $1 \mathrm{~b}$ using the Kaiser Raman Workstation, when the powder and the disc were removed and replaced six times, ranged from $0.1 \%$ relative standard deviation (RSD) for the most intense measurements (configuration D with $150-212 \mu \mathrm{m}$ aspirin) to $4.3 \%$ RSD for the weaker Raman signals (configuration A with $0-38 \mu \mathrm{m}$ Avicel). 


\section{Results}

\section{Effect of moving Raman active inter-layer through bulk powder}

When the sample configurations illustrated in Figure 1a were used to examine the effect of moving the sulphur disc through the different powders, the results shown in Figures 2 and 3 were obtained. The measured intensities are the average of 3 replicate measurements. Three main observations can be made about the results in Figures 2 and 3:

(1) For each powder, the transmitted Raman intensities obtained when the sulphur disc is at the top (P5 in Figures 1a, 2 and 3) or bottom (P1 in Figures 1a, 2 and 3) are similar, but lower than intensities obtained when the disc is within the powder; this arises from photon losses at the air-sample interface when the Raman active disc is at a surface position. ${ }^{1,17-19}$

(2) The sulphur Raman signal intensity at the outer and inner (2 $\mathrm{mm}$ of powder before the sulphur disc) positions differs by a factor of 1.5, 2.6 and 3.0 for Avicel with a particle size of $<38,53-106$ and $150-212 \mu \mathrm{m}$, respectively. This suggests that a greater portion of photons are lost at the air-sulphur disc interface as the particle size of Avicel increases; larger granular Avicel particles will pack less well than smaller particles and consequently, there are likely to be more air gaps at the Avicel-sulphur disc interface. The difference between the signal for the outer and inner positions is smaller for aspirin than for Avicel (2.4 compared to 3.0, respectively) of the same size range (150 $212 \mu \mathrm{m})$. This is likely to be due to differences in the packing characteristics of the

granular (Avicel) and needle (aspirin) particles. In a recent study ${ }^{18}$ of an inter-layer containing $\mathrm{TiO}_{2}$ within a stack of eight $\mathrm{MCC}$ discs, the $\mathrm{TiO}_{2}$ Raman signal from the inner compared to the outer positions was greater by a factor of 1.8. Although the particle size of the MCC used to make the discs was not given in the paper, fewer air gaps would be expected with a pressed MCC disc compared to a loose powder; hence, the factor of 1.8 is similar to that observed in the present study with the smallest $(<38 \mu \mathrm{m})$ particle size of Avicel. 


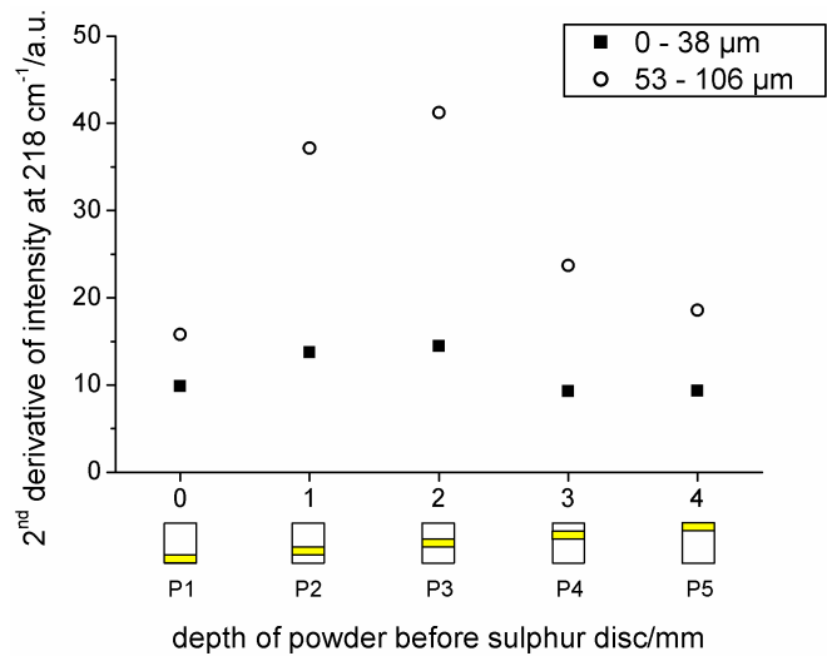

Figure 2. Change in the magnitude of the second derivative transmission sulphur signal at $218 \mathrm{~cm}^{-1}$ as the sulphur disc is progressively moved through $4 \mathrm{~mm}$ of Avicel particles with a size range of $0-38$ or $53-106 \mu \mathrm{m}$. 


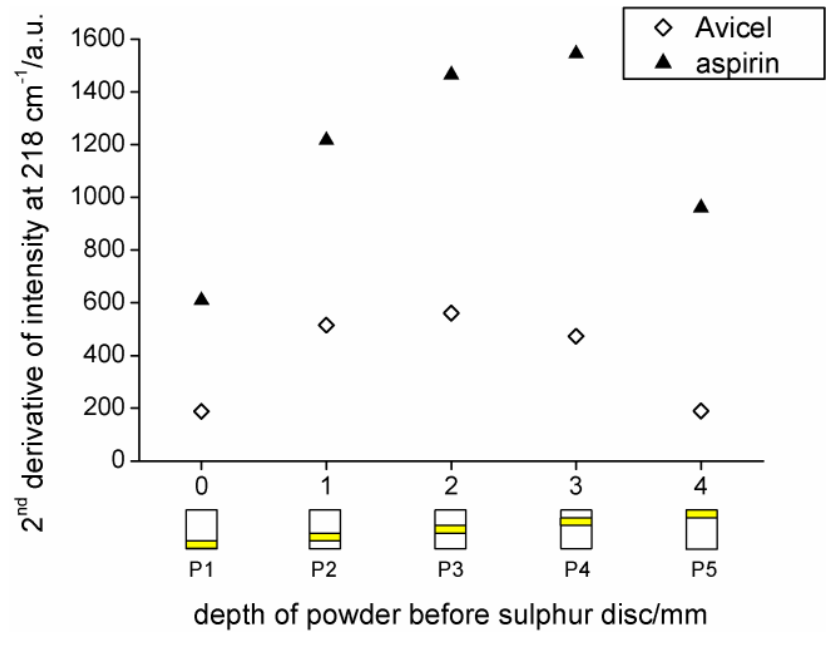

Figure 3. Change in the magnitude of the second derivative transmission sulphur signal at $218 \mathrm{~cm}^{-1}$ as the sulphur disc is progressively moved through $4 \mathrm{~mm}$ of Avicel or aspirin particles with a size range of $150-212 \mu \mathrm{m}$. 
(3) The size and type of the particles affect the magnitude of the transmission Raman signal. Light is more diffusely scattered by small particles ${ }^{11,21-22}$ and consequently, the transmitted Raman signal for sulphur increases when the laser and Raman photons propagate through Avicel of increasing particle size. However, aspirin particles attenuate the sulphur Raman signal less than Avicel particles of the same size $(150-212 \mu \mathrm{m})$. This could be due to differences in the particle shape and/or the absorption properties of the two materials.

The general shape of the plots observed in Figures 2 and 3 is consistent with those obtained in a recent study of an inter-layer containing $\mathrm{TiO}_{2}$ within a stack of eight $\mathrm{MCC}$ discs. ${ }^{18}$ However, it can also be seen that the type and size of the powder has a marked effect on the intensity of the transmitted Raman signal from the sulphur inter-layer; the effect of such factors was not investigated in previous studies. ${ }^{1,18-19}$

\section{Effect of bulk powder on the attenuation of the exciting laser intensity and the transmitted Raman intensity}

Measurement of the attenuation coefficients for laser and Raman photons. In this set of experiments, a Kaiser MkII probe was used to illuminate samples from above with a Kaiser PhAT probe beneath the sample to collect the transmitted Raman signal. The sample configurations illustrated in (i) and (ii) of Figure $1 \mathrm{c}(\mathrm{x}=0$ to 6$)$ were used to investigate the attenuation of the laser and Raman photons, respectively, by Avicel with a particle size of $150-212 \mu \mathrm{m}$. As the sample was illuminated from above, the laser photons passed through the Avicel prior to reaching the sulphur disc (except where $\mathrm{x}=0$ ) in Figure 1c(i). Consequently, the sulphur peak intensity in the transmission Raman spectrum depended on the attenuation of the laser photons by the powder. With the arrangements illustrated in Figure 1c(ii), the laser impinged on the sulphur disc first and so the Raman photons generated by the disc propagated through different thicknesses of Avicel. In this case, the measured sulphur peak intensity in the transmission Raman spectrum was dependent on the attenuating effects of the powder on the sulphur Raman photons. The sulphur intensities from both sets of experiments were normalised with respect to the sulphur intensity obtained when no powder was positioned above or below 
the disc, and the data are plotted in Figure 4. The attenuation coefficients associated with the propagation of laser and Raman photons can be derived from a plot of the natural logarithm of the sulphur signal intensity against Avicel depth. With the sample configurations illustrated in Figure 1c(i), there are likely to be differences in the extent of photon loss at the sample surface when the illuminated surface is the sulphur disc or Avicel powder. This problem does not arise for the second set of measurements as the illuminated surface is always the sulphur disc (Figure 1c(ii)). To ensure that the illuminated surface was the same within each data set, the first point in Figure $4(0 \mathrm{~mm}$ Avicel) was not included in the calculation of the attenuation coefficients. The remaining points were used to produce plots of the natural logarithm of the normalised sulphur intensities against Avicel depth. The slopes of the best fit line, corresponding to the attenuation coefficients for propagation of the laser and Raman photons, were $-0.9 \pm 0.01 \mathrm{~mm}^{-1}$ and $-0.9 \pm 0.02 \mathrm{~mm}^{-1}$, respectively. Hence, it can be concluded that the attenuation of the laser and Raman photons in Avicel powder of $150-212 \mu \mathrm{m}$ is the same.

Calculation of attenuation coefficients for laser photons. The results given in Figures 2 and 3 are a combined effect of the influence of the different powders on the propagation of the laser and Raman photons. By making an additional set of measurements using the Kaiser Raman Workstation with illumination of the samples from below and the sample configurations illustrated in Figure 1b, which measures attenuation of the Raman photons, it was anticipated that each powder's effect on the propagation of the laser and the Raman photons in the transmission measurements could be decoupled. From a comparison of Figures 1a and 1b, it is apparent that configurations $\mathrm{P} 1$ and $\mathrm{A}, \mathrm{P} 2$ and $\mathrm{B}$, $\mathrm{P} 3$ and $\mathrm{C}, \mathrm{P} 4$ and $\mathrm{D}$ and $\mathrm{P} 5$ and $\mathrm{E}$ are related in the sense that for each pair there is the same amount of powder on top of the Raman active disc. To estimate the attenuation of the exciting laser intensity by $\mathrm{x} \mathrm{mm}$ of powder (as in Figure 1d), the following equation can be used:

$$
\frac{\operatorname{Intensity}(\mathrm{E})}{\operatorname{Intensity}(\mathrm{x})_{\text {calc }}}=\frac{\operatorname{Intensity}(\mathrm{Z})}{\operatorname{Intensity}(\mathrm{P})} \quad \text { Equation } 1
$$




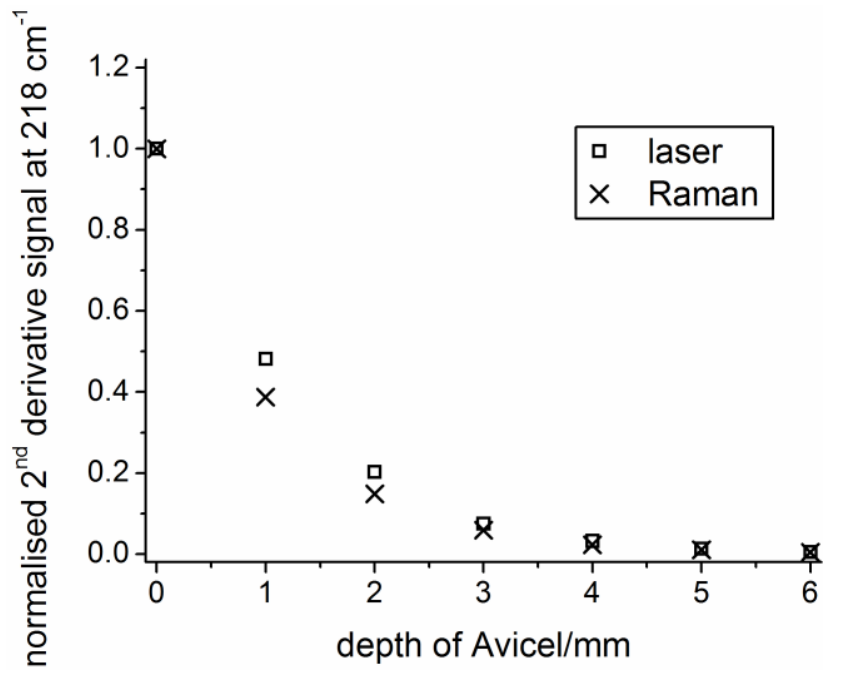

Figure 4. Characterisation of the propagation of laser and Raman photons via the normalised $2^{\text {nd }}$ derivative Raman signal at $218 \mathrm{~cm}^{-1}$ for sulphur through different depths of Avicel with a particle size of $150-212 \mu \mathrm{m}$. 
where Intensity $(\mathrm{P})$ is the measured sulphur signal intensity for configuration P1, P2, P3, $\mathrm{P} 4$ or $\mathrm{P} 5$, Intensity $(\mathrm{Z})$ is the measured sulphur signal intensity for configuration $\mathrm{A}, \mathrm{B}, \mathrm{C}$, $\mathrm{D}$ or $\mathrm{E}$, Intensity(E) is the measured sulphur signal intensity for configuration $\mathrm{E}$, and Intensity $(\mathrm{x})_{\text {calc }}$ gives the calculated sulphur signal intensity when there is a depth of $\mathrm{x} \mathrm{mm}$ of powder before the sulphur disc.

Calculation of the estimated sulphur intensities obtained for propagation of laser photons through different depths of $150-212 \mu \mathrm{m}$ Avicel powder prior to reaching the sulphur disc (i.e. Intensity $(\mathrm{x})_{\text {calc }}$ ) is given in the Supporting Information (See Tables S-1 to S-3 and Figure S-3). The calculated and measured sulphur signal intensities for the configurations depicted in Figures 1d ( $x=0$ to 4$)$ and $1 \mathrm{~b}$, respectively, can be used to compare the effects of the powder on the attenuation of the laser and the transmitted Raman signal, respectively. The results obtained for the propagation of laser and Raman photons through different depths of Avicel with a particle size of $150-212 \mu \mathrm{m}$ are shown in Figure 5. To aid comparison, the calculated (see Table S-3) and measured (see Table S-2) sulphur signal intensities were normalised by dividing by the value corresponding to $0 \mathrm{~mm}$ powder in each case, prior to plotting in Figure 5. There is a much bigger difference between the laser and Raman results at each depth of Avicel in Figure 5 than in Figure 4 (which was based on direct experimental measurements). This arises because application of Equation 1 does not take into account the greater photon losses that occur when the sulphur disc is at a surface position, which caused overestimation of Intensity $(\mathrm{x})_{\text {calc. }}$. However, for the data in Figure 5 corresponding to powder depths of 1 to $3 \mathrm{~mm}$, the calculations are consistent. So, by using this data and plotting the natural logarithm of the sulphur intensity against the Avicel depth, it was possible to obtain the attenuation coefficient associated with propagation of the laser photons through the powder. The attenuation coefficient for the Raman photons was calculated using the data in Figure 5 for powder depths of 1 to $4 \mathrm{~mm}$. When data corresponding to $0 \mathrm{~mm}$ of powder was included, a single linear model was not always appropriate owing to additional photon losses at the collection surface when the sample is a sulphur disc, which from previous studies is not unexpected. ${ }^{1,17-19}$ 


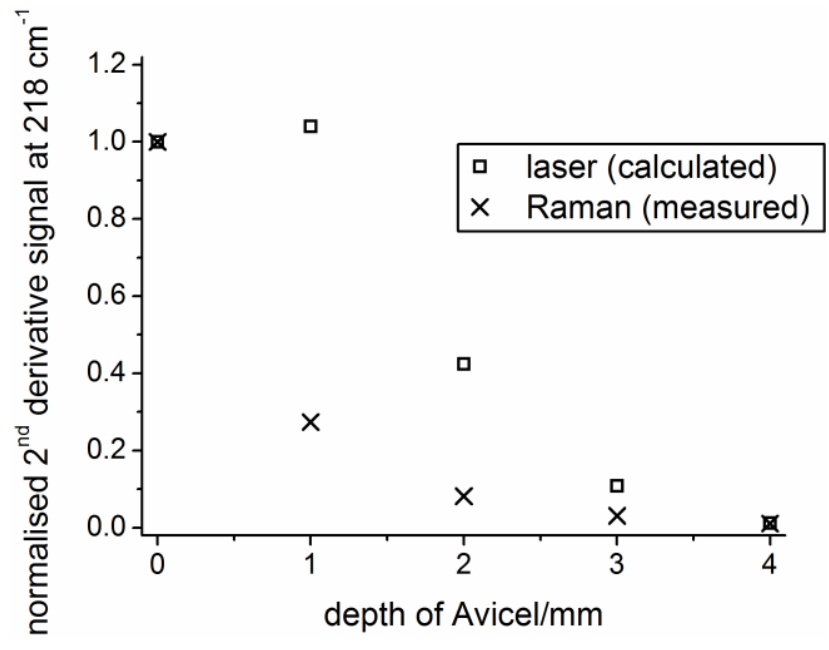

Figure 5. Plot comparing the propagation of laser and Raman photons via the calculated and measured $2^{\text {nd }}$ derivative Raman signals, respectively, for sulphur through different depths of Avicel with a particle size of $150-212 \mu \mathrm{m}$. 
The attenuation coefficients for the laser and Raman photons for Avicel particles of $150-212 \mu \mathrm{m}$, calculated from the slope of the best fit line, were $-1.1 \pm 0.14 \mathrm{~mm}^{-1}$ and $-1.1 \pm 0.03 \mathrm{~mm}^{-1}$, respectively. The values obtained are in reasonable agreement with those derived from Figure 4. The small difference between the two measured values for the Raman attenuation coefficient could be due to the different optical configurations (Kaiser Workstation and MkII/PhAT probe set-up) employed in the two sets of experiments. Similarly, the calculated laser attenuation coefficient might be expected to differ slightly to that measured in Figure 4. However, the key point is that the calculated laser attenuation coefficient is the same as the measured Raman attenuation coefficient, which validates the methodology employed. Consequently, the procedure was then used to calculate the attenuation coefficients for the other particle size ranges of Avicel and for aspirin of particle size $150-212 \mu \mathrm{m}$. The results obtained are given in Table 1 along with the values obtained previously for Avicel with a particle size of $150-212 \mu \mathrm{m}$.

Table 1 indicates that the values for the attenuation of laser radiation derived using Equation 1 are similar, within experimental error, to those measured directly for the attenuation of the Raman radiation for each individual powder. Therefore, if either material absorbs radiation then the levels of absorption of the laser and Raman photons must be similar; this is consistent with the observation of approximately symmetrical (about the sample centre) depth profiles in Figures 2 and $3 .{ }^{18}$ Previous numerical simulations and time-resolved measurements in backscatter ${ }^{15-16}$ and $\operatorname{transmission}^{17}$ modes of photon migration in powder samples consisting of a single Raman active material have shown that the Raman signal decays slower than the laser radiation. This is because Raman photons are generated throughout the depth of a Raman active sample as well as being diffusely scattered, in comparison to the laser photons that undergo diffuse scattering. In the present study, the sulphur Raman photons are generated in one plane of the sample and consequently, the attenuation coefficients for the laser and Raman photons are similar.

Table 1 shows that the attenuation coefficients for both the laser and Raman photons increase with a decrease in particle size. This is because smaller particles are more highly scattering than larger particles of the same material. ${ }^{11,21-22}$ Higher levels of 
Table 1. Attenuation coefficients ( \pm standard error) for the propagation of laser and Raman photons through Avicel and aspirin.

\begin{tabular}{|c|c|c|}
\hline Material & $\begin{array}{l}\text { Attenuation } \\
\text { coefficient for } \\
\text { laser photons } \\
\text { a } \\
/ \mathrm{mm}^{-1}\end{array}$ & $\begin{array}{c}\text { Attenuation } \\
\text { coefficient for } \\
\text { Raman photons }{ }^{\mathrm{b}} \\
/ \mathrm{mm}^{-1}\end{array}$ \\
\hline Avicel $0-38 \mu \mathrm{m}$ & $-2.1 \pm 0.23$ & $-1.9 \pm 0.05$ \\
\hline Avicel $53-106 \mu \mathrm{m}$ & $-1.9 \pm 0.22$ & $-1.6 \pm 0.07$ \\
\hline Avicel $150-212 \mu \mathrm{m}$ & $-1.1 \pm 0.14$ & $-1.1 \pm 0.03$ \\
\hline Aspirin $150-212 \mu \mathrm{m}$ & $-0.4 \pm 0.08$ & $-0.6 \pm 0.06$ \\
\hline
\end{tabular}

${ }^{\text {a }}$ Calculated using powder depths of $1-3 \mathrm{~mm}$

${ }^{\mathrm{b}}$ Calculated using powder depths of $1-4 \mathrm{~mm}$ 
photon attenuation were observed in Avicel than in aspirin of the same particle size (150 $-212 \mu \mathrm{m})$. This is likely to be due to differences in the particle shape and/or the absorption properties of the two materials. The attenuation coefficients calculated for Avicel are of a similar magnitude to that estimated from a study by Everall et al ${ }^{19}$ A value of approximately $-1.5 \mathrm{~mm}^{-1}$ was derived from the transmitted Raman signal for a poly(diacetylene) (PDA) crystal buried in the centre of a stack of MCC discs; the particle size of the MCC used to make the discs is not given in the paper. Also, separate terms were not measured for the decay of the laser and Raman photons and so the attenuation coefficient derived is a composite value.

As a plot of the natural logarithm of signal intensity against powder depth is linear with the slope equal to the attenuation coefficient, there will also be a logarithmic relationship between signal intensity and the attenuation coefficient. Therefore, the natural logarithm of the sulphur Raman signal obtained for the five sample configurations (P1 to P5) in Figure 1a was plotted against the Raman attenuation coefficient given in Table 1. Linear plots were obtained with the slope equal to the powder depth. The slopes ranged from 3.6 to $4.3 \mathrm{~mm}$ with an average value of $3.8 \mathrm{~mm}$, which is in excellent agreement with the actual powder depth of $4 \mathrm{~mm}$ employed in configurations P1 to P5. 


\section{Conclusions}

Transmission Raman measurements have been carried out on a sulphur disc at different positions in a $4 \mathrm{~mm}$ depth of powder. Lower signals were observed when the sulphur disc was placed at the top or bottom surface of the powder bed compared to positions within the bed, which is consistent with previous studies. ${ }^{1,18-19}$ However, the present study has also shown that the difference between the sulphur Raman signal intensity at the inner and outer positions is dependent on the particle size and type of the bulk powder.

The attenuation coefficient for propagation of Raman photons through different powders was determined by varying the depth of powder after the sulphur disc. From comparison of the signals obtained with those from experiments where the position of the disc was varied in $4 \mathrm{~mm}$ of powder, it was possible to calculate the attenuation coefficient for propagation of laser photons. The calculated attenuation coefficients were generally similar for laser and Raman photon propagation in each of the powders studied. The attenuation was lower for larger particle sizes of Avicel. In addition, Avicel attenuated laser and Raman photons more than aspirin of the same particle size.

In previous studies, the effects of physical properties were either not explored or considered to be less significant than for near infrared spectrometry. ${ }^{10}$ However, the present study has shown that the intensity of transmitted Raman spectra is affected by the particle size and type of the bulk powder. In some cases, it may be possible to reduce the effects of such factors on spectra through normalisation or other appropriate preprocessing. For the study of heterogeneous pharmaceutical dosage forms, the bias of the transmitted Raman signal towards the centre of the sample can be reduced through use of a photon diode at the illumination and collection surfaces. ${ }^{18,23}$ However, variations in the intensity of the transmitted Raman signal with particle properties have major implications for quantification of an analyte in powders where the composition and the physical properties of calibration and test samples are not matched. 


\section{Acknowledgements}

NT was supported by the Engineering and Physical Sciences Research Council and the Centre for Process Analytics and Control Technology (CPACT). The Royal Society is thanked for the award of a University Research Fellowship to AN.

Supporting Information Available: This material is available free of charge via the Internet at http://pubs.acs.org. 


\section{References}

(1) Matousek, P.; Parker, A. W. Appl. Spectrosc. 2006, 60, 1353-1357.

(2) Matousek, P.; Parker, A. W. J. Raman Spectrosc. 2007, 38, 563-567.

(3) Johansson, J.; Sparén, A.; Svensson, O.; Folestad, S.; Claybourn, M. Appl. Spectrosc. 2007, 61, 1211-1218.

(4) Eliasson, C.; Macleod, N. A.; Jayes, L. C.; Clarke, F. C.; Hammond, S. V.; Smith, M. R.; Matousek, P. J. Pharm. Biomed. Anal. 2008, 47, 221-229.

(5) Macleod, N. A.; Matousek, P. Pharm. Res. 2008, 25, 2205-2215.

(6) Sparén, A.; Johansson, J.; Svensson, O.; Folestad, S.; Claybourn, M. Am. Pharm. Rev. 2009, 12, 62-71.

(7) Aina, A.; Hargreaves, M. D.; Matousek, P.; Burley, J. C. Analyst 2010, 135, 2328-2333.

(8) Fransson, M.; Johansson, J.; Sparén, A.; Svensson, O. J. Chemom. 2010, 24, 674680.

(9) Hargreaves, M. D.; Macleod, N. A.; Smith, M. R.; Andrews, D.; Hammond, S. V.; Matousek, P. J. Pharm. Biomed. Anal. 2011, 54, 463-468.

(10) Buckley, K.; Matousek, P. J. Pharm. Biomed. Anal. 2011, 55, 645-652.

(11) Schrader, B.; Bergmann, G. Z. Anal. Chem. Fresenius 1967, 225, 230-247.

(12) Klosowski, J.; Steger, E. J. Raman Spectrosc. 1979, 8, 169-171.

(13) Eremeeva, T. P.; Vorsina, I. A. J. Appl. Spectrosc. 1976, 25, 1361-1364.

(14) Ferraro, J. R.; Ziomek, J. S.; Mack, G. Spectrochim. Acta 1961, 17, 802-814.

(15) Everall, N.; Hahn, T.; Matousek, P.; Parker, A. W.; Towrie, M. Appl. Spectrosc. 2001, 55, 1701-1708. 
(16) Everall, N.; Hahn, T.; Matousek, P.; Parker, A. W.; Towrie, M. Appl. Spectrosc. 2004, 58, 591-597.

(17) Everall, N.; Matousek, P.; Macleod, N.; Ronayne, K. L.; Clark, I. P. Appl. Spectrosc. 2010, 64, 52-60.

(18) Matousek, P.; Everall, N.; Littlejohn, D.; Nordon, A.; Bloomfield, M. Appl. Spectrosc. 2011, 65, 724-733.

(19) Everall, N.; Priestnall, I.; Dallin, P.; Andrews, J.; Lewis, I.; Davis, K.; Owen, H.; George, M. W. Appl. Spectrosc. 2010, 64, 476-484.

(20) Bellamy, L. J.; Littlejohn, D.; Nordon, A. Analyst 2008, 133, 58-64.

(21) Schrader, B.; Hoffmann, A.; Keller, S. Spectrochim. Acta 1991, 47A, 1135-1148.

(22) Bull, C. R. J. Mod. Opt. 1990, 37, 1955-1964.

(23) Matousek, P. Appl. Spectrosc. 2007, 61, 845-854. 
for TOC only

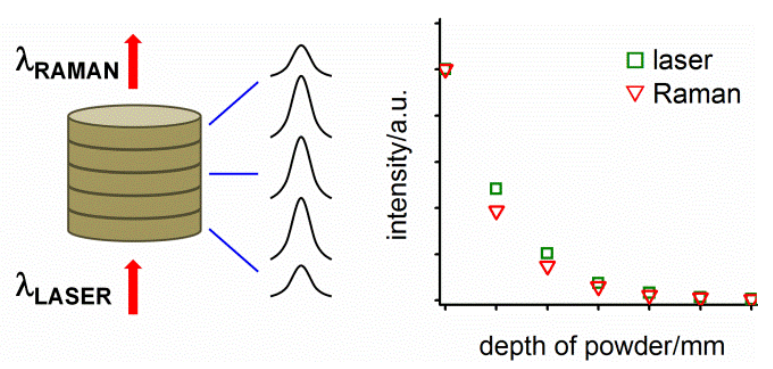

